CHAPMAN : SOME MAIN LINES OF ADVANCE, ETC.

\title{
Some Main Lines of Advance in the Domain of Modern Analytical Chemistry.
}

\section{A Lecture Delivered before the Chemical Society on March 15Th, 1917.}

\section{By A. Chaston Chapman.}

Analytical chemistry has often been referred to as the handmaiden of the other branches of our science, and whilst this was an entirely unobjectionable description in so far as it implied indispensable assistance, it was a little unfortunate in that it carried with it a certain suggestion of inferiority. From the dawn of scientific chemistry in the seventeenth century to a period within the recollection of a good many chemists who are still happily among us, analytical chemistry was almost synonymous with chemistry itself, and it is only in comparatively recent times that it has become a separate branch of applied chemistry with its own literature, its own aims, and its own specialised practitioners. Whilst the division of chemistry into various separated branches became inevitable with the enormous development of the science, and had its obvious conveniences, the progressive subdivision of work has not been without its drawbacks, and even its dangers. That it has conduced to a narrowness of outlook and to a mental onesidedness is undeniable, and it is becoming increasingly difficult always to maintain a just sense of proportion to view facts in their true perspective and to keep a firm hold on fundamental principles. Of all the various members of the body chemical, perhaps none suffered more at first by this process of subdivision than analytical chemistry. During the first half of the last century, chemical analysis occupied a very high position, since not only was it clearly the foundation-stone on which the whole chemical fabric was built, but almost every chemist of distinction practised it assiduously and devoted much of his time to a study of its problems. One need only recall in this connexion such names as those of Berzelius, Gay-Lussac, Marignac, Bunsen, Dumas, Stas, Liebig, and Wöhler. With the birth of modern organic chemistry and its colossal development during the past half-century - a development which, be it remembered, was largely dependent in the beginning on the analytical labours of GayLussac, Liebig, Dumas, and other early workers in the field-the analytical branch of our science was gradually relegated to a comparatively humble position. In the presence of this new and fertile field, in which every thrust of the spade served to bring 
to light some discovery of the highest importance and of the most absorbing interest, it is scarcely to be wondered at that the great majority of chemists should have forsaken the older branch, and that the all-important foundation-stone should have tended to become regarded more and more as merely the useful handmaiden. That the lamp of analytical chemistry was kept well alight during the period in question, notwithstanding the superior attractions of organic chemistry, is evidenced by the epoch-making researches of Bunsen and Kirchhoff on spectrum analysis, by numerous records of observations on the qualitative electrolytic decomposition of metallic salts, on which the important branch of electrochemical analysis was later on to be founded, chiefly by the labours of Classen and his students, and by the many investigations having for their object the perfection and simplification of methods of gas analysis. Last, but not least, I may mention in this connexion the establishment of the Fresenius Laboratory in Wiesbaden in 1848, and a few years later (1862) the foundation of the Zeitschrift für analytische Chemie, the first journal, I believe, which was devoted exclusively to the interests of analytical chemistry. Our own Journal, The Analyst, did not appear until 1877.

Great as is the temptation, it is not my intention to deal at any length with the history of analytical chemistry during the latter half of the nineteenth century. I will merely content myself with remarking that at a certain point in that history the rate of progress in its scientific development had slowed down so considerably that any chemist taking a superficial survey might well have been justified in supposing that future advances would be restricted to improvements in existing methods and to the extended application of those methods to the solution of new technical and industrial problems. That new lines of progress would be opened up must have seemed highly improbable, and it is recorded that a certain distinguished German chemist went so far as to utter the dictum that analytical chemistry presented no further problems. My task this evening will be to indicate briefly a few of the main lines along which the scientific development of modern analytical chemistry has proceeded, and to deal - however inadequately - with what I conceive to be the main causes of its restoration to something of its old position as a living and progressive part of our science. Foremost among these causes has unquestionably been the almost unparalleled growth during comparatively recent years of that younger member of the chemical family-I mean physical chemistry. That a proper understanding of analytical chemistry, as of every other department of the science, 
is dependent on a knowledge of those fundamental laws which it is the special province of physical chemistry to elucidate will appear to most chemists of to-day a self-evident proposition. Yet it was not until the publication of Ostwald's "Wissenschaftlichen Grundlagen der analytischen Chemie" in 1894 that this allimportant truth was presented to the chemical world, and not until some years later that it received anything like general recognition-at least in this country. To many generations of students chemical analysis had been taught as a useful art, consisting largely of a series of recipes which if followed conscientiously would usually lead to the desired result. Those recipes had often the authority of great names behind them, and so far as they went were admirable; but how many of those students were taught anything of the underlying principles, or were put by their teachers in a position to make for themselves such modifications of the orthodox processes as might at times become necessary? The result was to manufacture a body of more or less mechanical handworkers, and if, as fortunately happened, a number of these showed initiative and developed originality, it was in spite of, rather than in virtue of, the instruction they had received. This state of affairs was, however, inevitable, since, as Ostwald has pointed out, it was only with the advent of the general theory of chemical reactions and states of equilibrium that it became possible to elaborate a theory of analytical reactions and to place analytical chemistry on a really scientific foundation. It was then the beneficent work of physical chemistry to infuse new life into analytical chemistry, to help to place it on a firm and wide basis, and to do much to remove the reproach that this branch of chemical science, whilst representing a useful field of highly skilled manual labour, could scarcely be regarded as an intellectual occupation.

In three main directions this revivifying influence has made itself felt during the past twenty years. In the first place, it has supplied the explanation of a vast number of facts which had been arrived at empirically, and are of the greatest importance to the analyst; in the second place, it has greatly stimulated and directed original research, and, finally, it has enriched analytical chemistry with a number of new appliances and has resulted in the introduction of many new methods of inquiry.

Of all the theories of modern physical chemistry, none has had so far-reaching and important an effect on the development of analytical chemistry as those connected with the nature of solution. It is not, in fact, going too far to say that the general adoption of these theories and their application to analytical pro- 
cesses was the means of placing analytical chemistry for the first time on a really scientific basis. The years 1885,1886 , and 1887 , which for chemists will ever be memorable for the publication of the great generalisations of van't Hoff and Arrhenius, may justly be regarded as dates of outstanding importance in the history of analytical chemistry. To deal with this aspect of the subject at any length before an audience of chemists is quite unnecessary. That the theory of electrolytic dissociation has not met with universal acceptance is of little importance from this point of view, for I believe that even those chemists who regard it with the greatest disfavour would be inclined to admit that no other theory is able to explain so much or is capatle of welding the whole chaotic mass of analytical phenomena into such a coherent and harmonious whole.

The manner in which it has contributed to the building of a serviceable, if not complete, theory of indicators, and its helpfulness in explaining the phenomena of electrolysis, hydrolytic dissociation, and mass action, are too well known to need more than a passing reference.

As a further instance of the fruitfulness of this concept and of its value to analytical chemistry I may mention the application of the conductivity method to the determination of the point of neutrality of liquids. This method, first suggested by Küster and Grüters in 1903, has since received a considerable amount of attention at the hands of a great many workers, and the numerous contributions to the subject by Sörensen, Michaelis, and others represent a storehouse of very valuable information in connexion with the applicability of certain indicators to special purposes, the preparation of solutions of standard reaction, and the electrometric measurement of hydrogen-ion concentration. This work, which is undoubtedly capable of further development, has already proved of very great value in the investigation of certain biochemical problems, and will, no doubt, find extended application in the wider field of technical analysis.

In further exemplification of the close and fruitful connexion between physical chemistry and analytical chemistry, I may just refer to the vast amount of work which has been done during comparatively recent years in connexion with the colloidal state of matter and in the study of catalytic phenomena.

As showing, moreover, how some of the more recondite physical properties of matter may prove to be of practical importance to the analyst, I may perhaps be permitted to refer to the papers which $I$ have published alone or in collaboration with $H$. D. Law on the reducing action of hydrogen in its relation to analytical 
processes. In those papers it has been shown that much depends on the nature of the metallic surface from which the hydrogen is evolved. Thus, certain specimens of zinc which, owing to the presence of small quantities of impurities, are useless for the purpose of detecting and estimating minute traces of arsenic by the modified Marsh-Berzelius method, can usually be made suitable by coating the zinc with pure cadmium, thus producing a surface from which the hydrogen is evolved in a state of higher chemical activity.

In the same way, when an electrolytic method of estimating traces of arsenic is employed, much greater sensitiveness is obtained by using a lead or a cadmium rather than a platinum cathode. It is, of course, tolerably certain that the reducing efficiency of hydrogen, whether obtained by the interaction of metal and acid or by an electrolytic process, is dependent upon a number of factors, partly chemical and partly physical, and that the reactions are, in point of fact, very complex. There can, however, be very little doubt that among these factors the question of "potential" or "overvoltage" plays a prominent and important part.

The effect of employing cathodes of different metals in the reduction of arsenious oxide is shown by the following results:

\begin{tabular}{|c|c|c|c|c|c|}
\hline \multicolumn{2}{|c|}{ Cathode. } & & $\begin{array}{l}\text { Arsenious } \\
\text { oxide } \\
\text { added. }\end{array}$ & $\begin{array}{l}\text { Arsenious } \\
\text { oxide } \\
\text { found. }\end{array}$ & $\begin{array}{l}\text { Arsenious } \\
\text { oxide } \\
\text { left in flask }\end{array}$ \\
\hline Lead $\quad .$. & & & 10 & 10 & - \\
\hline Cadmium & $\ldots$ & $\ldots$ & 10 & 10 & - \\
\hline Tin $\quad \ldots$ & $\ldots$ & $\ldots$ & 10 & 10 & - \\
\hline Copper ... & $\ldots$ & $\ldots$ & 100 & 10 & 90 \\
\hline $\begin{array}{ll}\text { Silver } \quad \ldots \\
\end{array}$ & $\ldots$ & $\ldots$ & 50 & 10 & 40 \\
\hline Nickel $\ldots$ & $\ldots$ & $\ldots$ & 40 & 20 & 20 \\
\hline Platinum ( & & $\ldots$ & 1,000 & 0 & 1,000 \\
\hline Iron $\quad \ldots$ & $\ldots$ & $\ldots$ & 50 & 5 & 45 \\
\hline
\end{tabular}

From this it will be seen that even the overvoltage of metals-a subject which at first sight might appear to be mainly of academic interest-has, in fact, a very direct and important bearing on some of the everyday operations of the analytical laboratory.

Time forbids that I should deal at any greater length with this side of the intimate relationship existing between these two branches of our science, and I will now refer briefly to the service rendered by physical chemistry in the direction of increasing the analyst's equipment and of multiplying his means of attack.

To the balance, the microscope, and the spectroscope there have been added from time to time a number of other physical instruments, some of which have revolutionised many of the older branches of analytical chemistry and have even been the means of giving birth to new ones. Whilst in its cruder forms the 
polariscope goes back to the beginning of the last century, or even beyond, the modern polarimeter is of comparatively recent birth, and since the introduction of the Jellet instrument about 1860, the labours of physicists and opticians have been almost continuously devoted to the perfecting of this highly important analytical appliance. Without it, carbohydrate analysis would to-day have been in a very different position, and many of the problems which confront the analyst in connexion with the sugar, starch, confectionery, essential oil, and brewing industries would have been impossible of solution. In addition to these main applications of the polarimeter, there are many others of less importance, and new ones without doubt remain to be discovered.

Next perhaps in importance comes the refractometer, an instrument which finds wide and ever-increasing application in the analytical laboratory. What the polarimeter is to carbohydrate analysis the refractometer is to the analysis of fats and oils, and its assistance has been successfully invoked in the examination of brewing materials, milk serum, sugars, methyl and ethyl alcohols, glycerol, and many other natural and artificial products.

The calorimeter in all its forms and with all its modern refinements, as well as the various mercurial, resistance, and thermoelectric heat-measuring instruments, represent another section of what, for want of a better term, may be called analytical procedure, for the development of which the chemist is chiefly indebted to the physicist.

In addition to these physical instruments of almost everyday use, passing reference may be made to modern spectrographic apparatus, appliances for the determination of electrical conductivity, instruments for the measurement of colour and of turbidity, and, lastly-and of most recent introduction-apparatus suitable for making estimations of radioactivity.

I think I have said enough to show the general nature of the relationship existing between physical and analytical chemistry, and to indicate one of the main lines along which the latter branch of our science has advanced during comparatively recent years. Such indeed is the intimacy of the connexion that, as new discoveries are made by physical chemists, they will almost inevitably be utilised in the consolidation or extension of that great field of analytical chemistry in which so many of us are proud to labour.

I will now turn for a brief space to another side of my subject. If organic chemistry by its rapid growth, vast importance, and allcompelling interest was responsible for the temporary neglect of analytical chemistry at one stage of its history, it has since made 
ample amends by furnishing the analytical armoury with a number of very important weapons. If it has presented to the analyst innumerable problems of the highest importance, it has also supplied in many cases the means for their solution. To speak more plainly, it has been found during recent years that many of the more or less complex products of the organic laboratory are, in fact, very valuable analytical reagents, and that by their use old problems may often be better solved and new ones successfully attacked.

One of the earliest of these was phenylhydrazine, by the discovery of which in $1878 \mathrm{E}$. Fischer placed in the hands of chemists a reagent which is now almost as necessary in the analytical as in the purely organic laboratory. In qualitative analysis, it is only necessary to refer to the application of the osazone reaction to the identification of the various sugars, a problem the difficulty of which is only equalled by its importance, whilst in quantitative analysis it has a large sphere of usefulness in the estimation of aldehydes and in the quantitative examination of compounds containing the carbonyl, nitroso-, and nitro-groups. In addition to phenylhydrazine itself, considerable use has been found for some of its substituted derivatives, such as $s$-phenylmethylhydrazine, $s$-diphenylhydrazine, and the $p$-bromo- and $p$-nitro-compounds. In this connexion, reference may also be made to $\beta$-naphthylhydrazine, a substance which Ekenstein and Lobry de Bruyn have shown to be of great service in the recognition and separation of certain carbohydrates and for the estimation of vanillin, and to semicarbazide, a substance which has proved so useful in the isolation and identification of the aldehydic and ketonic constituents of many essential oils.

As further examples of organic compounds which have been successfully applied to the identification and estimation of organic substances of technical importance, mention may be made of phloroglucinol, digitonin, picric acid, and picrolonic acid. The estimation of pentoses and pentosans in certain foodstuffs and in many agricultural products is often a matter of importance, and, as is well known, the method almost invariably adopted consists in distilling the substance under investigation with hydrochloric acid and estimating the furfural in the distillate. For this purpose, phloroglucinol, which forms with furfural a sparingly soluble compound-the so-called phloroglucide-is often made use of. In the same way, methyl-pentoses and methyl-pentosans can be estimated as the corresponding phloroglucinol compound. Further than this, the difference in the solubility in alcohol of the two phloroglucides affords a means for the approximate separation of 
the pentoses (for example, arabinose and xylose) and the methylpentoses (for example, rhamnose and fucose).

In 1910 Windaus made the discovery that one molecule of cholesterol is capable of uniting with one molecule of digitonin to form a compound of high molecular weight $\left(\mathrm{C}_{82} \mathrm{H}_{140} \mathrm{O}_{29}\right)$ which is very insoluble, and can consequently be conveniently employed for the estimation of the former substance- a matter of considerable importance to physiological and occasionally to technical chemists. Since digitonin also forms a similarly insoluble compound with phytosterol, it has been employed for the purpose of obtaining either or both of these alcohols in a pure condition prior to converting them into their acetates, as in the ordinary Bömer test. In this way, the detection of vegetable oils in oils of animal origin, or vice versa, is much facilitated.

The extensive use made of picric acid for the identification, and occasionally for the estimation, of alkaloids and other bases is too well known to need more than a passing reference, and more recently picrolonic acid (dinitrophenylmethylpyrazolone) has been employed for the same purpose. The picrolonates are in some cases less readily soluble than the corresponding picrates, crystallise well, and are often very characteristic. The importance of adding to our means of identifying such substances as arginine, histidine, lysine, guanidine, and other physiologically important bases will be obvious to all who have followed the developments of modern biochemistry.

It will be seen that the instances $I$ have given above are all cases in which one organic compound has been made use of for the identification or estimation of another. I will now refer to a more interesting and, in a sense, more significant application of certain organic compounds, namely, to the detection and estimation of what may be conveniently described as the simpler inorganic acids and bases.

One of the earliest of these is the $m$-phenylenediamine test for nitrous acid, for which we are indebted to Peter Griess. Already in 1870 Griess had recommended the use of one of the diaminobenzoic acids for the detection of nitrous acid, and in 1878 he proposed the use of the above reagent as being much more sensitive, and the method was worked out on the quantitative side about the same time by Preusse and Tiemann. In the following year Griess published a further paper, in which he recommended for the same purpose the use of a mixture of sulphanilic acid and a-naphthylamine-a method which is occasionally ascribed to Ilosvay, who merely recommended the substitution of acetic acid for a mineral acid as the acidifying medium. 
Another well-known colorimetric method for the detection and estimation of minute quantities of nitrous and nitric acids is due to Lunge, and involves the employment of diphenylamine. Although, unfortunately, not specific, it has proved of the greatest service in the estimation of minute traces of nitrogen acids in sulphuric acid, milk, and other technical products.

A good many other organic compounds, such as phenolsulphonic acid and resorcinol, have been recommended at various times for the detection and estimation of nitrates and nitrites, but it will not be necessary to refer to these in any detail. It will, of course, be readily understood that the nature of my subject-even if time permitted-renders it unnecessary for me to deal by way of illustration with more than a few of the instances in which organic compounds have been pressed into the service of this branch of general analytical chemistry, and I shall confine my attention to those methods which have been very thoroughly tested and have been elevated to the rank of standard processes.

Whilst dealing with the estimation of commonly occurring inorganic acids, mention may be made of the benzidine method for sulphuric acid and the use of "nitron" for the estimation of nitric acid. The former method, which owes its origin to W. Müller, depends on the fact that benzidine sulphate is almost entirely insoluble in cold water in the presence of an excess of benzidine hydrochloride, and since the method is a volumetric one, it possesses the advantage of rapidity.

Notwithstanding that it has been studied and recommended for special purposes by such experienced workers as Raschig and G. v. Knorre, it is obviously very unlikely that any organic compound will ever replace barium as a general reagent for the estimation of sulphuric acid. I have referred to this method rather in the hope that it may stimulate investigation in this direction, since the occurrence of an insoluble sulphate of an organic base, and still more the discovery of an insoluble nitrate, such as is referred to below, render it probable that similar insoluble salts, suitable for analytical purposes and possibly representing some well-defined advantages over existing methods, still remain to be discovered.

In 1905, in the course of an investigation of the endo-iminotriazoles, Busch observed that these bases are characterised by the formation of very sparingly soluble nitrates. By a fortunate chance, it happened that the most readily prepared of these compounds, namely, $1: 4$-diphenyl-3:5-endo-anilo-4:5-dihydro-1:2:4triazole, was the one which gave a nitrate possessing the highest degree of insolubility. One molecule of this base unites with one molecule of nitric acid, giving a compound of the formula 
$\mathrm{C}_{20} \mathrm{H}_{16} \mathrm{~N}_{4}, \mathrm{HNO}_{3}$, and it is capable of giving a precipitate in a solution containing as little as 1 part of nitric acid in 80,000 parts of water.

This base, which can be obtained commercially under the more easily remembered and more euphonious name "nitron," is very easily employed as a reagent, and furnishes us for the first time with a means of making a direct gravimetric estimation of nitric acid. It has been applied with success to the estimation of nitrates in water and in a considerable number of commercial products, such as natural nitrates, nitrocellulose, soils and plants, and is particularly well suited for the estimation of nitrates in liquids containing much organic matter. It has also been found that the method is applicable to the estimation of picric acid, since 1 part in 250,000 parts of water gives a precipitate of the "nitron" picrate. The discovery of unexpectedly useful properties, such as the insolubility of this nitrate-and many other striking examples might easily be quoted-adds a great attraction to the study of pure, synthetic organic chemistry.

I will now pass for a few moments to the application of organic compounds to the quantitative separation and estimation of many of the commoner and some of the rarer metals.

One of the first substances to be used for this purpose, and one which is susceptible of somewhat wide application, is nitroso- $\beta$ naphthol, which has been very thoroughly studied by G. v. Knorre and his colleagues. It has been found, for example, that by the use of this reagent nickel may be separated from cobalt; iron from aluminium; copper from cadmum, magnesium, manganese, zinc, mercury, and lead; iron from manganese, zinc, nickel, and chromium; iron from glucinum; copper, iron, and cobalt from antimony and arsenic; and iron from zirconium.

Many of these separations can be effected with ease and with accuracy, the metallic derivatives of the nitroso- $\beta$-naphthol being easily dealt with, and being, of course, readily converted by ignition into the corresponding oxides. The copper compound has the formula $\left(\mathrm{NO} \cdot \mathrm{C}_{10} \mathrm{H}_{6} \cdot \mathrm{O}\right)_{2} \mathrm{Cu}$.

$m$-Nitrobenzoic acid has been successfully used for the quantitative separation of thorium from cerium, lanthanum, and didymium in the analysis, for example, of monazite sands, whilst palladium can be readily separated from platinum and other metals of the platinum group by means of acetylene.

De minimis non curat may be true of the law, but it certainly is not true of modern chemistry, which in certain directions is almost coming to be regarded as the chemistry of traces. The importance to the physiological chemist, to the metallurgical 
chemist, and to the food chemist of being able to detect with certainty small traces of substances is well recognised, and peculiar interest therefore attached to Tschugaev's observation in 1905 that dimethylglyoxime constitutes a delicate test for nickel. $\mathrm{He}$ showed, in fact, that it was capable of detecting 1 part of the metal in more than 1,000,000 parts of water, and of affording certain indications, even when cobalt was present to the extent of 5,000 times that of the nickel. The reaction may be represented by the following equation:

$$
2 \mathrm{C}_{4} \mathrm{H}_{8} \mathrm{O}_{2} \mathrm{~N}_{2}+\mathrm{NiCl}_{2}+2 \mathrm{NH}_{3}=\left(\mathrm{C}_{4} \mathrm{H}_{7} \mathrm{O}_{2} \mathrm{~N}_{2}\right)_{2} \mathrm{Ni}+2 \mathrm{NH}_{4} \mathrm{Cl} \text {. }
$$

This method has been very thoroughly investigated by many chemists, some of whom have introduced modifications with the object of increasing its sensitiveness. Thus, it has been quite recently stated that, by the adoption of certain modifications, as little as 0.02 milligram can be readily detected in 50 c.c. of solution, whilst some years ago Armit and, Harden stated that they were able, when working with pure nickel sulphate, to detect as little as 0.001 milligram in 30 c.c., whilst 0.003 milligram gave a very marked pink colour in that volume of liquid.

As a reagent for the colorimetric estimation of traces of nickel, dimethylglyoxime possesses very great and obvious advantages over the ammonium sulphide method. The coloration is more characteristic, traces of iron do not interfere, and the method is, of course, much more sensitive.

As a means of estimating nickel in alloys, particularly in the presence of cobalt, zinc, and iron, this method has proved exceedingly useful, being rapid and possessed of a tolerably high degree of accuracy.

Another oxime, namely, $a$-benzildioxime, originally described by Tschugaev, has been recommended by Atack for the qualitative detection of nickel and for its estimation. It forms an intensely red nickel compound having the formula $\mathrm{C}_{28} \mathrm{H}_{22} \mathrm{O}_{4} \mathrm{~N}_{4} \mathrm{Ni}$, containing only 10.9 per cent. of nickel. It is even more sensitive than dimethylglyoxime, and it has been stated that with this reagent 1 part of nickel in 10 million parts of water can be detected. Like dimethylglyoxime, it has been recently utilised for the detection of traces of nickel in hardened edible fats, in which nickel has been used as a catalyst. When working on 50 grams of the fat, 1 part of nickel in 5 million parts of the fat can be detected. It is of interest to note, in passing, that the $\beta$-isomeride does not yield the nickel reaction.

Another organic compound utilised during recent years for the estimation of nickel is dicyanodiamidine. This reagent, which was originally recommended by Grossmann in 1906, is used in the form 
of the sulphate, and the nickel compound, which is a well-crystalline, yellow substance, has the composition $\mathrm{Ni}\left(\mathrm{C}_{2} \mathrm{H}_{5} \mathrm{ON}_{4}\right)_{2}, 2 \mathrm{H}_{2} \mathrm{O}$. By the use of this reagent, nickel may be separated from cobalt, iron, chromium, zinc, and other metals, and, given suitable conditions, may be estimated with a high degree of accuracy. It has, in fact, been used very largely, particularly in Continental laboratories, for the analysis of German silver, commercial nickel, nickel steel, and other alloys. Although it is not by any means as sensitive as either of the two above-mentioned reagents, it is said to be capable of detecting 0.5 milligram of nickel in the presence of as much as 1 gram of cobalt.

The above methods for the estimation of nickel are of great technical importance, since the precipitates are crystalline and form readily at the ordinary temperature. They can be filtered with ease, they are of constant composition, and the percentages of nickel are not high. The employment of these more or less complicated organic reagents is, as I have indicated, of comparatively recent introduction, and it is not too much to hope that similarly serviceable methods will be discovered for the separation and for the rapid and accurate estimation of other closely allied elements.

In 1909 Baudisch discovered that the ammonium salt of nitrosophenylhydroxylamine $\left(\mathrm{C}_{6} \mathrm{H}_{5} \cdot \mathrm{N}[\mathrm{NO}] \cdot \mathrm{ONH}_{4}\right)$ was capable of being used for the quantitative precipitation of iron and of copper, and for the separation of these metals from a number of others with which they are frequently associated in practice. This substance, to which the simpler name "cupferron" has been applied, has been found to be capable of somewhat wide application, and has proved a valuable addition to our laboratory reagents.

Ferric iron is precipitated completely in cold solutions containing hydrochloric, sulphuric, or acetic acids as the compound $\left(\mathrm{C}_{6} \mathrm{H}_{5} \cdot \mathrm{N}[\mathrm{NO}] \cdot \mathrm{O}\right)_{3} \mathrm{Fe}$, and may readily be separated from aluminium, chromium, and indeed from most other of the common metals, including, by a special process, even copper.

The method has proved useful for the separation and estimation of iron in a number of commercial products, and R. Fresenius, who has submitted it to a critical study, states that the separation of iron and aluminium can be carried out more conveniently and with greater accuracy by the use of this substance than by any other gravimetric method.

I do not propose to enter into any details of the various separations effected by this reagent, but will confine myself to pointing out that by its aid copper may easily be separated from cadmium, zinc, and many other metals, and that both titanium and zirconium 
may be separated from iron and from aluminium. The titanium compound is a bright yellow substance having the formula $\left(\mathrm{C}_{6} \mathrm{H}_{5} \cdot \mathrm{N}[\mathrm{NO}] \cdot \mathrm{O}\right)_{4} \mathrm{Ti}$.

As an example of the degree of accuracy which may be reached in these separations, it may be pointed out that, when quantities of iron varying from 0.03 gram to 0.3 gram were precipitated in the presence of quantities of aluminium and chromium equal to fifty times the weight of iron present, the error rarely exceeded \pm 0.2 milligram. I mention this detail merely for the purpose of showing that in the case of these complex organic reagents, the many advantages which are frequently obtained are not necessarily secured at the expense of accuracy.

I think I have dealt at sufficient length with this division of my subject, and I will now turn for a few moments to the consideration of a third main line of advance, namely, that concerned with the utilisation of what may be conveniently called biological methods. In the future we may succeed in synthesising enzymes and even precipitins, but that day is not yet, and for some time to come we must remain dependent on the activity of the living organism for the reagents to which $\mathrm{I}$ am now referring.

With the mechanism of enzyme action we are not now concerned. What is of importance from the analytical point of view is its specific character. To such an extent is this the case that the enzymes are actually capable of discriminating between certain of the carbohydrates and their optical isomerides. Thus, $d$-glucose, $d$-mannose, $d$-fructose, and $d$-galactose are fermentable by yeast, whilst their optical isomerides are unfermentable.

In order that a given sugar other than the four above mentioned may be fermented, it is essential that the yeast employed should contain the enzyme necessary for its conversion into one or other of those hexoses. Now yeasts of different species do not all contain the same enzymes, and it happens, therefore, that a certain species of yeast may be capable of fermenting one carbohydrate and incapable of fermenting another.

Of the various enzymes, invertase is one of the most widely distributed among the saccharomycetes, and consequently the great majority of yeasts are capable of fermenting sucrose. On the other hand, lactase occurs in only a comparatively small number of species, and consequently a great many yeasts, including the ordinary brewers' yeast, are incapable of fermenting lactose.

The following table may be of interest as showing at a glance the behaviour of certain of the yeast species towards several of the more commonly occurring sugars : 
$\begin{array}{ccccc} & \text { Dex- Fruc- Man-Galac- Malt. Sucr- } & \text { Lac- } \\ \text { Yeast. } & \text { trose. tose. nose. tose. ose. ose. } & \text { tose. }\end{array}$

Sacch. cerevisiae ...

Sacch. cerevisiae, Carlsborg

Sacch. Pastorianus

Sacch. ellipsoideus

Sacch. Marxianus ..

Sacch Trioi

Sacch. anomalus $\cdots \quad \cdots$

$\begin{array}{lll}\text { Sacch. fragilis } & \cdots & \cdots \\ & \cdots & \cdots\end{array}$

Kefir

$\begin{array}{ll}+ & + \\ + & + \\ + & + \\ + & + \\ + & + \\ + & + \\ + & + \\ + & +\end{array}$

+
+
+
+
+
0
+
+
+
+

+
+
+
+
+
+
0
0
+
0

+
+
+
+
0
0
0
0
0
0

+
+
+
+
+
+
+
+
+

0

0

0

0

0

0

0

$+$

The sign + indicates that the yeast in question is capable, and the sign 0 that it is incapable, of bringing about fermentation.

The secretion of any particular enzyme appears to be a very constant attribute of a given species, and it -has not been found possible by varying the nature of the food supply or the general environment of a given species to cause it to secrete other enzymes than those normally present. It is this constancy of enzyme production and this selective character that render certain of the yeast species so useful to the analyst, enabling him to arrive at the composition of complex carbohydrate mixtures, the analysis of which would be impossible by any other means.

The method is clearly one which must be applied with caution, and it demands, moreover, some biological training on the part of the operator. The technique, however, is not difficult, and at the present day the majority of analytical chemists, particularly those who are concerned with the analysis of foodstuffs, realise that a certain amount of training in elementary bacteriology is a necessary part of their professional equipment.

It will, of course, be clear that, in addition to yeasts, other organisms which secrete enzymes, such as moulds, may be utilised for analytical purposes, and the preparation of Taka-diastase from Aspergillus oryzae is an illustration in point. Torulæ, again, have recently been pressed into the service, since some of these, unlike the yeasts, do not contain any invertase, and so are capable of fermenting away dextrose and fructose, leaving sucrose unattacked. Biological methods such as I am now referring to are in everyday use in the analysis of many sugar products, such as commercial glucoses and invert-sugars, and find extensive employment in the analytical examination of complex carbohydrate mixtures such as many of the prepared foods intended for infants and for invalids.

As one further example of the usefulness of this method, it may be pointed out that, whilst the top yeast as obtained in English breweries converts raffinose into fructose and melibiose, the bottom yeast as obtained in Continental breweries, which contains melibiase as well as invertase, converts it into fructose, galactose, and 
dextrose, that is to say, into completely fermentable products. It is therefore possible by employing these two types of yeast, and by making a simple polarimetric observation, to estimate the amount of raffinose present in a mixture of sugars-a problem which only a few years ago would have been considered impossible of solution.

Another "analytical" method of a more definitely biological character, but one which has already shown itself to be of great practical importance, is that depending on the "precipitin" reaction-a reaction which permits of the identification of so-called homologous proteins and their differentiation from others which they resemble so closely that they are indistinguishable by purely chemical means. The principle underlying the method is that if a solution of any given protein be injected into the blood of an animal, the blood serum of that animal will produce a precipitate with an infusion containing the particular protein injected, but not with any other. Thus, if horse-blood serum be injected into a rabbit, the serum of the rabbit's blood will produce a precipitate when added to an extract of horseflesh, but not with an extract of any other kind of flesh. It thus becomes possible by means of this method to identify horseflesh in mixed foodstuffs, such as sausages. It is also possible to detect small quantities of castor seeds in feeding cakes, to distinguish between hen-egg albumin and the albumin of the eggs of other birds, between the milk of one animal and that of another, between genuine and artificial honey, and even, so it is stated, between the seeds of two-rowed and of six-rowed barley. The importance of the reaction for the identification of human blood in medico-legal investigations is well recognised.

I have now touched on a few of the main lines along which analytical chemistry has advanced during recent years, and have endeavoured to show that, so far from being the exhausted and lethargic handmaiden, it is, in fact, as alive, as progressive, and as originative of research as any other branch of our science. That this is not always fully realised, and that there has been-and is even now in some quarters-a tendency to regard analytical chemistry merely as a useful art and its practitioners as highly skilled labourers, is unhappily the case. That there are here and there a few analysts to whom that description might be correctly applied is undoubtedly true, but the same is, of course, equally true of the medical and other professions.

The analytical chemist of to-day is, in fact, being continually faced with new problems that frequently demand for their solution the possession in a high degree of those special qualities of 
intellect and character which go to make the successful investigator in the domain of pure chemical science. For many chemical consultants life is a continuous series of technical problems, and I am not indulging in any exaggerated language when $I$ say that the really successful consulting and analytical chemist must not only have a good general scientific training, an extensive knowledge of general chemistry, and a genuine love of his work, but he must be mentally alert and adaptable and possess the aptitude for research in a high degree.

With regard to the teaching of analytical chemistry, there is much that I should like to say if time permitted, and in particular I should like to plead again for the establishment in our universities and university colleges of chairs of analytical chemistry. I have already dealt with this subject at some length in an address to another society, and perhaps I may be allowed, in concluding, to quote the following remarks from that address:

"Having very briefly touched on the nature and extent of the scientific equipment needed for the successful practice of analytical chemistry, we may reasonably inquire whether the training which our young professional chemists obtain is such as is calculated to insure the best results. Whilst there may possibly be some difference of opinion as to the precise position which a study of chemical analysis should take in the training of the chemical teacher, there can surely be none as to its supreme importance in the training of the professional chemist. In the great majority of cases it is the actual instrument by which, directly or indirectly, he is to earn his livelihood, and in every case it must tend to produce (if properly taught as a living subject and not as a mass of tedious prescriptions and formulas) a deeper insight into the nature of chemical reactions, an appreciation of the influence of mass and other disturbing factors, and a recognition of the importance of attention to minute detail. In addition to this, it affords endless opportunity for the acquirement of dexterity in constructing and manipulating scientific appliances, and in all these ways renders invaluable service in the making of the successful technical chemist. Now, if all this be true - and I do not see how it can be denied-analytical chemistry ought clearly to take an outstanding position in our universities and university colleges, as it is from them that, more often than not, the young chemist proceeds directly to the practice of his profession. Unfortunately, the position which it takes in those institutions is not, as a rule, a high one, nor one at all commensurate with its importance. I believe I am correct in saying that in no university in this country does a chair of analytical chemistry exist, and that a subject which is 
admittedly of such great importance is entrusted to teachers who, however well qualified and capable they may be, have, as a rule, to teach it, if I may use the expression, incidentally. . . .

"So large a subject, and one which is in constant process of development, might well, it seems to me, be entrusted to a specially appointed professor, who would have the opportunity of keeping himself fully abreast of the developments of his subject, and who would have the time to deal with it in a manner practically impossible under the existing conditions. Such chairs of analytical chemistry exist in very many of the more important American and Continental universities, and it can scarcely be contended that what has been found desirable in so many other parts of the civilised world is unnecessary in Great Britain. Chairs of analytical chemistry, for example, exist in Yale, Virginia, Johns Hopkins, Cornell, and Columbia Universities, to name only those of which I know, and I believe I am correct in saying that in Columbia University there are no fewer than three such professors. In many of the university prospectuses great emphasis is laid on the importance of analytical chemistry, and from one of the Yale calendars I cannot refrain from quoting the following words: "There is probably no branch of chemical study as important as qualitative analysis in its use in developing the reasoning faculties and enabling the student to generalise and to classify chemical phenomena.' In Heidelberg, Munich, Leipzig, Würzburg, and other German universities, in the Imperial technical high schools at Stuttgart, Vienna, and elsewhere, such chairs exist, as well as at Upsala, in most of the Swiss and Belgian universities, and in some of the Italian. In regard to France and one or two other countries I have no definite information, but I think I have said enough to establish my point-that in many of the world's leading universities the teaching of analytical chemistry is entrusted to a specially appointed professor, who takes equal academic rank with his other chemical colleagues. Even when this is not the case, assistant professors or special assistants are frequently appointed to deal solely with this branch of chemistry. It clearly cannot be objected that it has not been our custom in this country to appoint professors to deal with special branches of chemistry, since in some of our colleges chairs exist devoted to physical chemistry, biochemistry, tinctorial chemistry, fuel chemistry, brewing chemistry, agricultural chemistry, technical chemistry, and metallurgy.

"I am not foolish enough to imagine that the establishment of chairs of analytical chemistry in all or any of our universities and colleges would bring forth a new heaven or a new earth, but 
at least it is certain that this highly important branch of chemistry would be taught under better conditions than those which in many cases exist at present. The teachers, being in a position to devote themselves entirely to their special branch of instruction, would be able to give more time and attention to the student, and surely in no branch of chemistry is close and constant supervision of practical work so necessary. They would also have the time to make themselves thoroughly conversant with their subject in both its theoretical and practical aspects, as well as to keep in touch with modern developments, and their laboratories might even become in process of time centres of original work in a department of our science in which research has been for so long neglected."

Since the above was written, some signs of progress auguring well for the future have been observable. That analytical chemistry is in a condition of vigorous growth $I$ have endeavoured to show, and that it may continue to flourish exceedingly must be the earnest wish of every member of this Society. All the branches of our science are reciprocally reactive and closely interdependent, and on the progress of British chemistry as a whole, and on the position which it holds in the national esteem, the future of this country, and indeed of the Empire itself, will to no small extent depend. May our rulers and our people be wiser in the future than they have been in the past. 